\title{
High-Sensitivity Ozone Sensing Using 280 nm Deep Ultraviolet Light-Emitting Diode for Detection of Natural Hazard Ozone
}

\author{
Yoshinobu Aoyagi ${ }^{1}$, Misaichi Takeuchi ${ }^{1}$, Kaoru Yoshida ${ }^{1}$, Masahito Kurouchi ${ }^{1}$, Tsutomu Araki ${ }^{2}$, \\ Yasushi Nanishi ${ }^{2}$, Hiroyasi Sugano ${ }^{3}$, Yumi Ahiko ${ }^{4}$, Hirotaka Nakamura ${ }^{5}$ \\ ${ }^{1}$ Global Innovation Research Organization, Ritsumeikan University, Shiga, Japan; ${ }^{2}$ Faculty of Engineering Science, Ritsumeikan \\ University, Shiga, Japan; ${ }^{3}$ Hikari Technology, Hikari, Japan; ${ }^{4}$ Miuraori Lab., Tokyo, Japan; ${ }^{5}$ Tokyo Metropolitan Industrial Tech- \\ nology Research Institute, Tokyo, Japan. \\ Email: aoyagi@fc.ritsumei.ac.jp
}

Received February $22^{\text {nd }}, 2012$; revised April $3^{\text {rd }}, 2012$; accepted May $13^{\text {th }}, 2012$

\begin{abstract}
Recently ozone is one of natural hazards which comes from cars, industry using ozone for sterilization of organic and inorganic materials and for water purification. So, ozone sensing becomes very important, and convenient and accurate ozone sensor is required. A new high sensitivity ozone sensing system using an deep ultra-violet light emitting diode (DUV-LED) operated at the wavelength of $280 \mathrm{~nm}$ has been successfully constructed. The fabrication of diode operated at $280 \mathrm{~nm}$ is much easier than that of DUV-LED operated at Hg lamp wavelength of $254 \mathrm{~nm}$. The system is compact and possible to sense the ozone concentration less than $0.1 \mathrm{ppm}$ with an accuracy of $0.5 \%$ easily with low power DUV-LED of around 200 micro Watts operated at $280 \mathrm{~nm}$ without any data processing circuit.
\end{abstract}

Keywords: Ozone Sensing; Deep Ultra Violet Light Emitting Diode; DUV-LED; High Sensitivity; Long Life; Compact

\section{Introduction}

The importance of ozone sensing has recently increased, because ozone is harmful gas which is generated from industrial use of ozone for sterilization and cleaning of biomaterials and semiconductor devises and also water sterilization. Furthermore, ozone is produced in the surface air by photochemical reactions involving unburned fuel vapors and nitrogen oxides produced at high temperatures by car engines. So, the depletion of ozone is very important. So, continuous, high-sensitivity easy detection of ozone is indispensable.

Ozone sensing is currently conducted by monitoring the conductance change in thin-film-like semiconductors containing $\mathrm{ZnO}[1,2], \mathrm{In}_{2} \mathrm{O}_{3}$ [3,4]. Previous studies have tried to measure ozone using a galvanic technique [5]. These devices have the advantages of being compact and very cheap, but their lifetime is less than one year and their accuracy is very low at $\pm 50 \%$. Ozone sensing using a Hg lamp was used for the purpose of solar-blind sensing in the aerospace industry [6,7]. Researchers have also applied UV light to the chemiluminometric measurement of atmospheric ozone [8]. There have been attempts to use optical fibers for sensing [9]. However, there are few reports on the determination of ozone concentration in the air using Hg lamp systems, because of the difficulties in accurate system construction with a high sensitivity suitable for ozone monitoring and with a long life that would provide an accuracy of approximately $\pm 0.5 \%$. An attempt was made to use a long-pass absorption chamber to achieve high sensitivity [10]. However, the cost of this system was very high and it was unstable. The life of the Hg lamp was only about one year and the size of the system was much larger than conventional sensors. Moreover, the Hg used in the lamp is a harmful metal, which has potentially detrimental environmental affects.

We recently developed a new type of ozone sensing system using a low-power deep ultra violet light emitting diode (hereafter DUV-LED) operated at the wavelength of $280 \mathrm{~nm}$ as a light source, instead of an Hg lamp. This system has the advantages of being very compact, having a long lifetime, and being free from any harmful materials.

In this report, we present our experimental results and demonstrate the utility of this system.

\section{Experiments and Discussion}

Figure 1 shows the typical spectra of a $\mathrm{Hg}$ lamp and DUV-LED operated at the wavelength of $280 \mathrm{~nm}$. The 

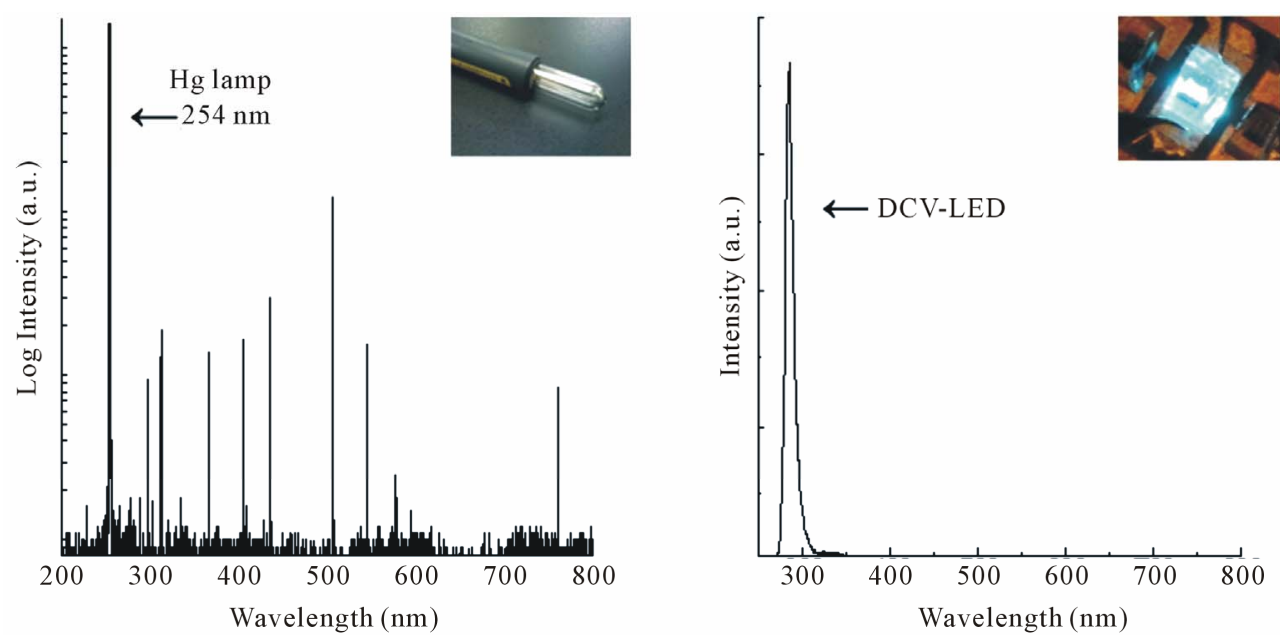

Figure 1. Spectrum of Hg lamp and DUV-LED operated at $280 \mathrm{~nm}$.

size of $\mathrm{Hg}$ lamp is at least a few centimeters and the lamp needs high voltage, whereas the size of the DUV-LED is only $500 \mu \mathrm{m}$ and the voltage necessary is only 10 or 20 volt. The Hg lamp emits light at various wavelengths, whereas the DUV-LED only emits light at a single wavelength of $280 \mathrm{~nm}$. In case of DUV-LED, any wavelength in DUV region can be chosen, depending on any different gases that might need to be monitored in addition to ozone. Our case the wavelength of $280 \mathrm{~nm}$ was chosen because the LED quantum efficiency at this wavelength region is much higher than that of LED operated at the wavelength of $\mathrm{Hg}$ lamp as shown in Figure 2. The big advantages to use the diode operated at the wavelength of $280 \mathrm{~nm}$ is easiness of the device fabrication process and high device quality because of lower concentration of Al in $\mathrm{AlGaN}$ component than that operated at $254 \mathrm{~nm}$. The larger the $\mathrm{Al}$ content in $\mathrm{AlGaN}$, which is a main material conducting DUV-LED, the lower the crystal quality and conductance between electrode and AlGaN are. The LED was fabricated by ourselves using metal organic chemical vapor phase epitaxy crystal growth technique.

Figure 3 shows the absorption band of ozone gas. There are several main bands, which are referred to as the Hartley band, Chappuls band, and the Wulf bands. Light absorption occurs with each band, depending on wavelength of light used. Depending on the requirements, a longer absorption band can be used. In our experiment, the absorption band around $260 \mathrm{~nm}$ was used. However, the wavelength actually used in our experiment was 280 $\mathrm{nm}$, because the external quantum efficiency of DUVLED was about 10 times larger than that at $254 \mathrm{~nm}$ of the main peak of the Hg lamp. However, the absorption coefficient of ozone at $280 \mathrm{~nm}$ is not very different from that at $254 \mathrm{~nm}$. Thus, the total system efficiency using the wavelength of $280 \mathrm{~nm}$ as ozone sensor, including diode quantum efficiency and absorption coefficient of

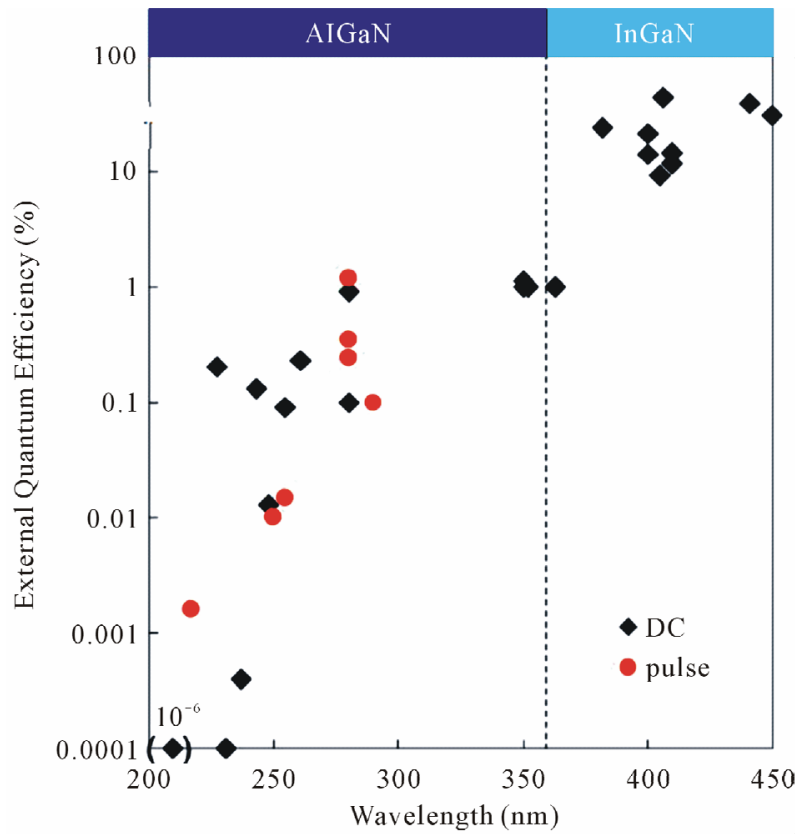

Figure 2. External quantum efficiency for various wavelengths of DUV-LED collected from papers published.

the light for ozone band, is much higher than the case using a $254 \mathrm{~nm}$ DUV-LED. In addition to this easy fabrication of the device of the $280 \mathrm{~nm}$ wavelength, the life time is also much longer of more than ten time compared to the $254 \mathrm{~nm}$ DUV-LED because of high quality materials for lower concentration of AlGaN. Actually the life time LED operated in blue region is more than 70,000 hours which is much beyond the life of the Hg lamp. At this moment the lifetime of DUV-LED is not so long compared to blue LED but it is expected to have such a long life near future. At this moment the lifetime is about $5000 \mathrm{hrs}$ if it will be operated at a few $\mathrm{mW}$ but it will be operated a few hundreds micro watt the life is expected 
to beyond easily $10,000 \mathrm{hrs}$.

Figure 4 illustrates the ozone sensing principle of DUV-LED. Light emitted from the DUV-LED propagates a monitoring chamber with a length of $20 \mathrm{~cm}$, which is significantly shorter than the conventional length of a $50 \mathrm{~cm}$ monitoring chamber. In this figure the wavelength of light source of Hg lamp of $255 \mathrm{~nm}$ was written but in case of DUV-LED the wavelength was 280 $\mathrm{nm}$. The gas sequence is shown in the same figure. The sample gas and zero gas, which are completely filtered

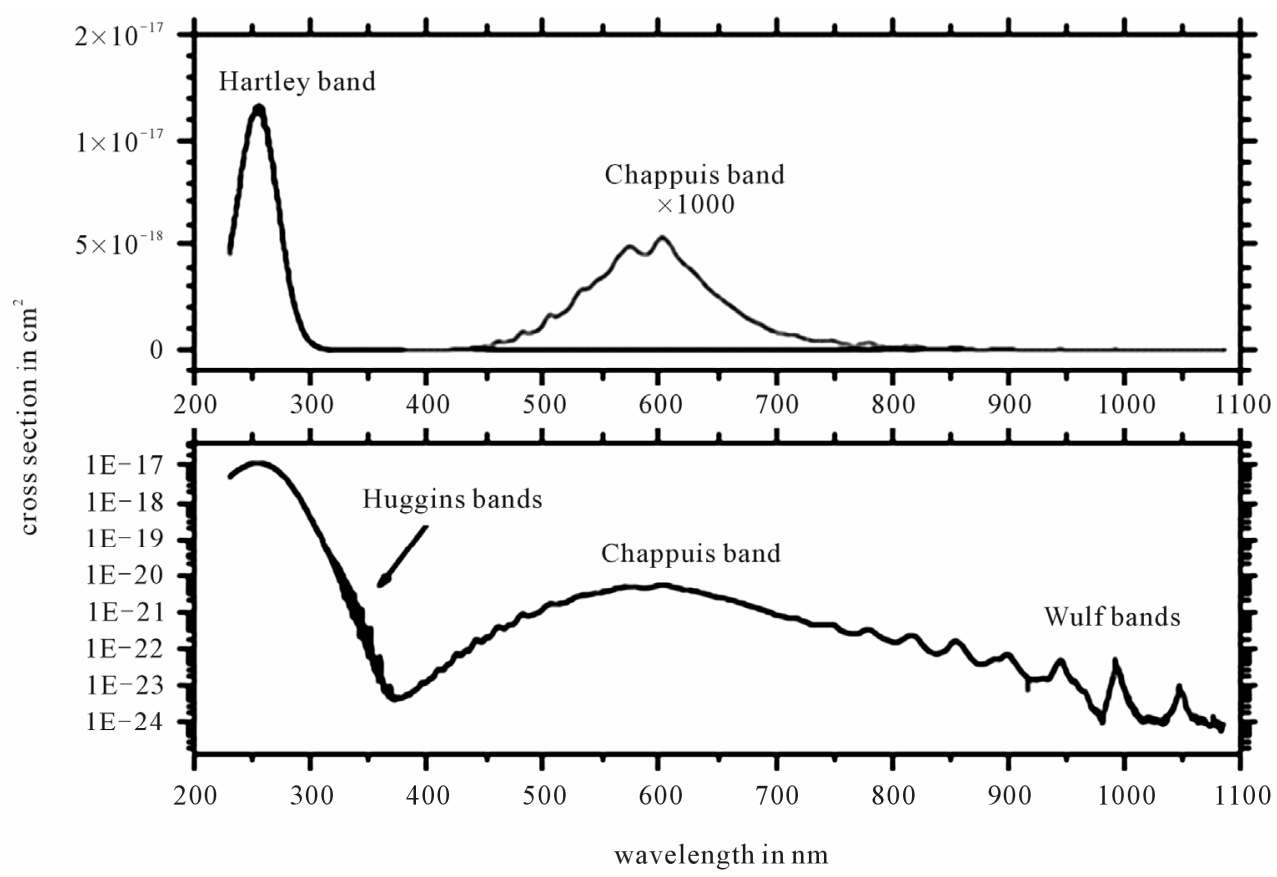

$0.27 \pm 0.03$

Figure 3. Ozone absorption cross section.
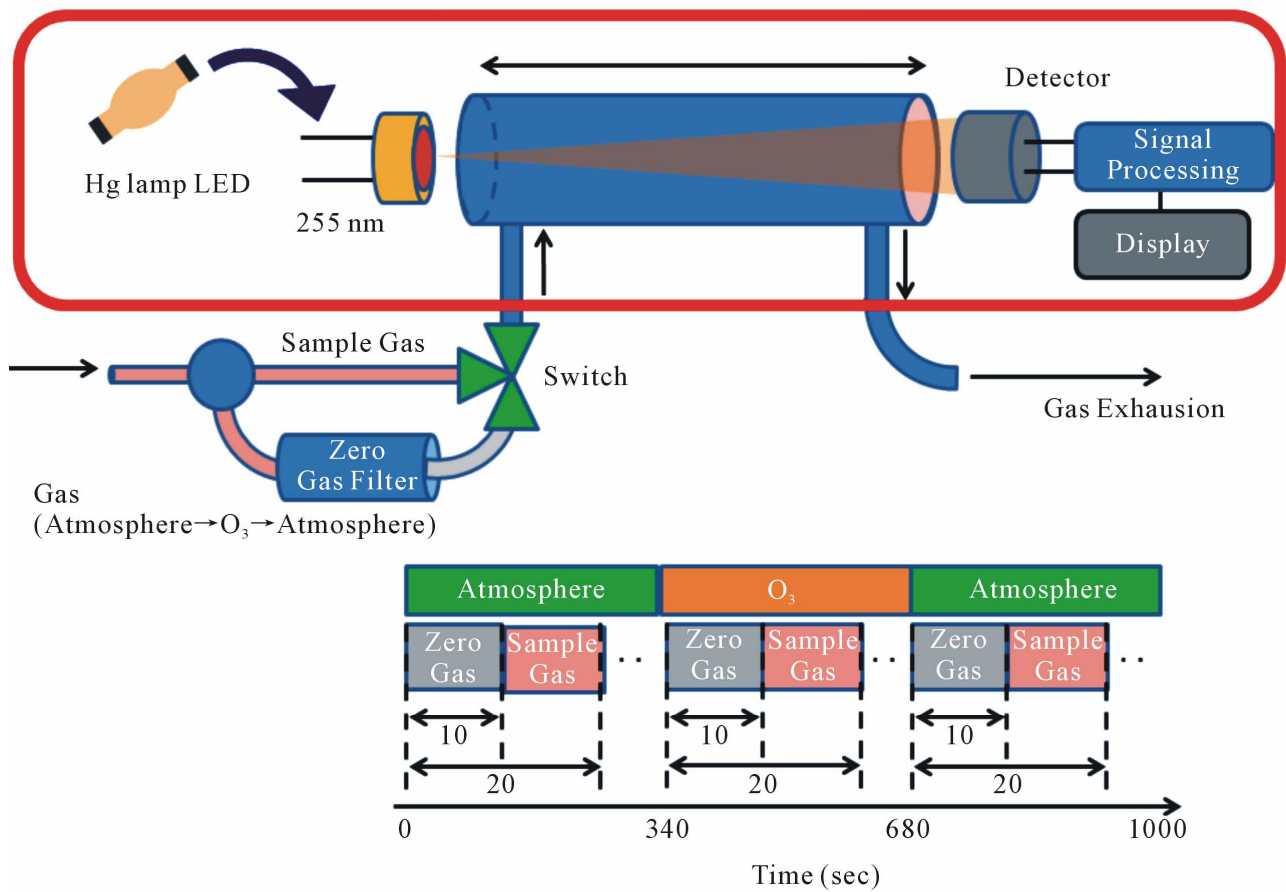

Figure 4. Schematic diagram of ozone sensing system used. The zero gas and sample gas are alternatively fed into absorption cell and estimated to evaluate ozone concentration. 
by a zero-gas filter, are alternately fed into the monitoring chamber at a set interval, until exhaustion. The light emitted from the DUV-LED is absorbed in the monitoring chamber and detected by an $\mathrm{AlGaN}$ detector. The experiment has been done at RT, and the detected signal was amplified using low noise amplifier. As a reference of zero gas monitoring we used zero gas filter made by a company. The zero gas filter has a capability of the reduction of ozone concentration of $1 / 20000$ when the inlet gas concentration of $1 \mathrm{ppm}$. So, this ozone filter is enough to use zero gas standard for our experiment within experimental error. So, the zero gas of ozone after through the filter is guaranteed. In this system at the beginning stage of 340 seconds the background ozone in atmosphere was observed. After the observation of background of ozone the ozone at monitoring position was observed $340 \mathrm{sec}$ and this cycle are repeated to get accurate date. Finally the ozone concentration at the monitoring point is estimated by this measurement. Figure $\mathbf{5}$ shows raw data of monitoring results of ozone levels of 0.19 and $0.08 \mathrm{ppm}$ under $200 \mu \mathrm{W}$ power DUV-LED light source and a short monitoring chamber of $20 \mathrm{~cm}$. This data suggests that the ozone concentration less than 0.1 ppm can easily be monitored by this short chamber and low power of light source condition of $280 \mathrm{~nm}$. The concentration of $0.1 \mathrm{ppm}$ of ozone is the recommended ACGIH-TLV-TWA value proscribed in the United States as the safe limit for human ozone exposure. Figure 6 shows the output signal intensity as a function of ozone concentration. Each point is an average of ten points sampling. Ozone concentration is changed by ozone generator and the ozone concentration was calibrated by other ozone monitoring system with the accuracy of $0.5 \%$. In our system we clearly find the sensitivity of ozone is much less than $0.1 \mathrm{ppm}$ and is near $0.01 \mathrm{ppm}$ and the linearity of the detected signal as a function of ozone concentration was confirmed. The accuracy of ozone concentration of the observation system is $\pm 0.5 \%$ as shown in this figure. If we increase the DUV-LED power to $2 \mathrm{~mW}$, which is now commercially available, the detection limit will be improved much more.

\section{Conclusion}

We demonstrated a high-sensitivity ozone sensing system using a low-power DUV-LED operated at $280 \mathrm{~nm}$

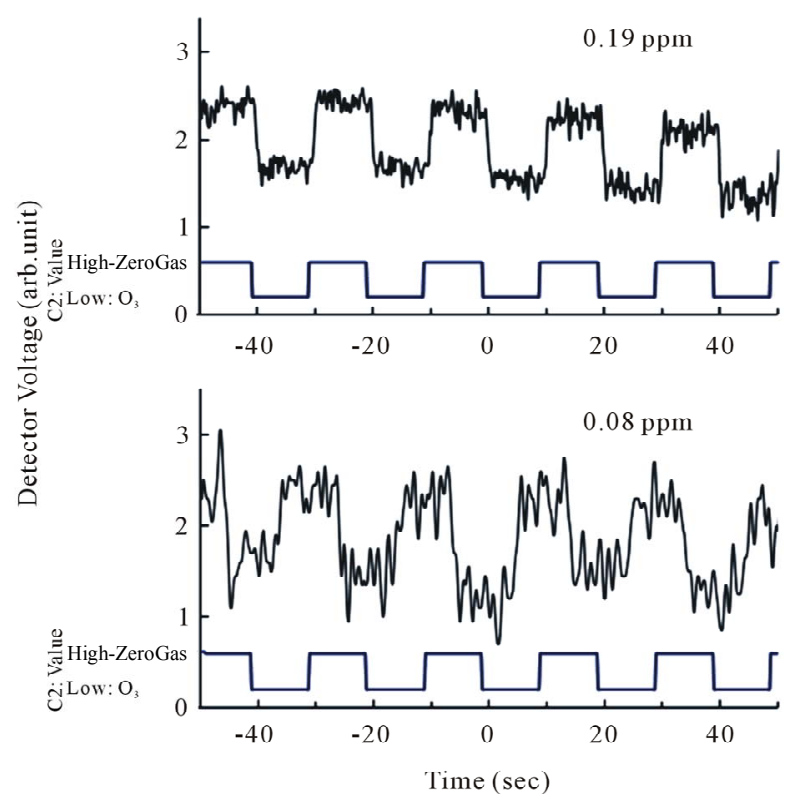

Figure 5. Row data of signal under ozone gas and ozone free gas with a concentration of $0.19 \mathrm{ppm}$ and $0.08 \mathrm{ppm}$.

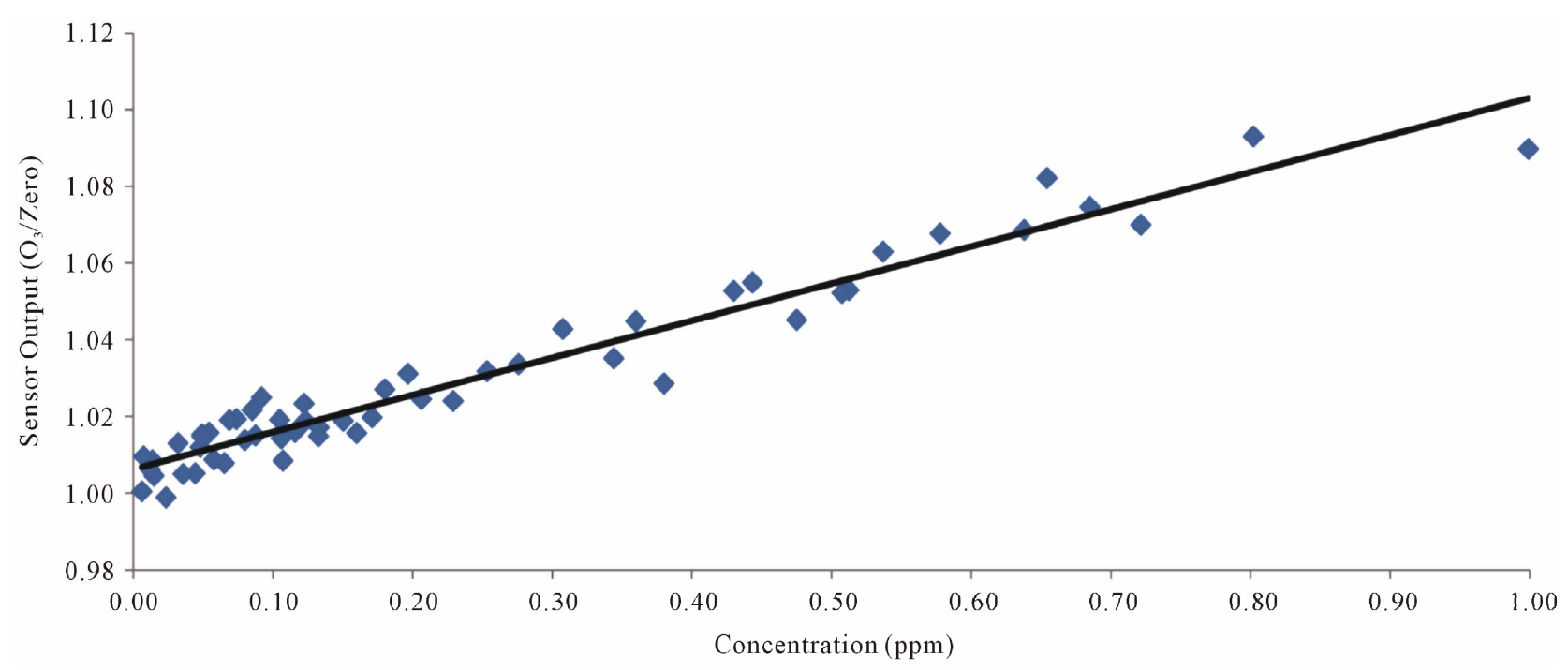

Figure 6. Photo-sensing output signal as a function of ozone concentration. 
but not $254 \mathrm{~nm} \mathrm{Hg}$ lamp light line. The sensitivity was much less than $0.1 \mathrm{ppm}$ and the accuracy of $0.5 \%$. This system is convenient and the size is small compared to the ozone sensing system using Hg lamp. In addition the proposed wavelength $280 \mathrm{~nm}$ in this paper as DUV-LED is very useful from the points of expectation of long lifetime of light source and high reliability and low voltage operation. This system will contribute to depletion of ozone in nature generated from various reasons, including industry, car, etc.

\section{REFERENCES}

[1] G. Kenanakis, D. Vernardou, E. Koudoumas, G. Kiriakidis and N. Katsarakis, "Ozone Sensing Properties of $\mathrm{ZnO}$ Nanostructures Grown by the Aqueous Chemical Growth Technique," Sensors and Actuators, Vol. B124, No. 1, 2007, pp. 187-191.

[2] M. Suchea, N. Katsurakis, S. Christoulakis, S. Nikolopoulou and G. Kiriakidis, "Low Temperature Indium Oxide Gas Sensors," Sensors and Actuators B: Chemical, Vol. 118, No. 1-2, 2006, pp. 135-141. doi:10.1016/j.snb.2006.04.020

[3] J. Li, Q. Li, J. V. Dyke and P. K. Dasgupta, "Atmospheric Ozone Measurement with an Inexpensive and Fully Automated Porous Tube Collector-Colorimeter," Talanta, Vol. 74, No. 4, 2008, pp. 958-964. doi:10.1016/j.talanta.2007.08.002

[4] R. Frycek, M. Jelinck, T. Kocourek, P. Fitl, M. Vrnata, V.
Myskik and M. Vrbova, "Thin Organic Layers Prepared by MSPLE for Gas Sensor Application," Thin Solid Films, Vol. 495, No. 1-2, 2006, pp. 308-311. doi:10.1016/j.tsf.2005.08.178

[5] S. Suzuki and K. Nagashima, "A Galvanic Solid-State Sensor for Monitoring Ozone and Nitrogen Dioxide," Analytica Chimica Acta, Vol. 144, 1982, pp. 261-266. doi:10.1016/S0003-2670(01)95542-4

[6] E. Trakhovsky, "Ozone Amount Determined by Transmittance Measurements in the Solar-Blind Ultraviolet Spectral Region," Applied Optics, Vol. 24, No. 21, 1985 , pp. 3519-3522. doi:10.1364/AO.24.003519

[7] L. M. Garrison, D. D. Doda and A. E. S. Green, "Total Ozone Determination by Spectroradiometry in the Middle Ultraviolet," Applied Optics, Vol. 18, No. 6, 1979, pp. 850-855. doi:10.1364/AO.18.000850

[8] T. Takayanagi, X.-L. Su, P. K. Dasgupta and R. W. Shaw, "Chemiluminometric Measurement of Atmospheric Ozone with Photoactivated Chromotropic Acid," Analytical Chemistry, Vol. 75, No. 21, 2003, pp. 5916-5925.

[9] S. O'Keeffe, C. Fitzpatrick and E. Lewis, "An Optical Fiber Based Ultra Violet and Visible Absorption Spectroscopy System for Ozone Concentration Monitoring," Sensors and Actuators B: Chemical, Vol. 125, No. 2, 2007, pp. 372-378. doi:10.1016/j.snb.2007.02.023

[10] E. M. Weinstock, C. M. Schiller and J. G. Anderson, "In Situ Stratospheric Ozone Measurements by Long Path UV Absorption: Developments and Interpretation," Journal of Geophysical Research, Atmospheres, Vol. 91, No. D4, 1986, pp. 5237-5248. doi:10.1029/JD091iD04p05237 